\title{
Development of an Extra Heavy Oil Filed in the Orinoco delta belt - A Viscous Challenge
}

\author{
E. Mus, Total.
}

The presentation will encompass the Petrocedeño field development in the Orinoco delta belt from reservoir characterization through to reservoir development strategy and management featuring some key learnings and on the fly adjustments that were required.

The huge oil accumulations present in the Orinoco belt exhibits current recoveries below $2 \%$ in the four areas developed so-far, typical of a low maturity, high stakes basin with a steep learning curve.

So far the local authorities have certified more than $250 \mathrm{~Gb}$ of reserves and one of the early and major developments to date is Petrocedeño, located in Junin region, production started in Dec. 2000.

To understand the challenges and potential for development optimization some key figures will be described:

The field carries Oil volumes in place close to $30 \mathrm{~Gb}$ OIP of $7 / 8^{\circ} \mathrm{API}$. Thanks to a reservoir temperature of $45^{\circ} \mathrm{C}$, the oil is mobile in the reservoir with a viscosity range of 2.000 to $4.000 \mathrm{cP}$.

It is producing 150 to $200 \mathrm{kbopd}$ under primary production since 2001 with an accumulated production of $500 \mathrm{MMb}$ as of May 2010. The production is feeding an Upgrader dedicated to and operated by Petrocedeño.

A total of 36 clusters with horizontal wells (average drain length of $1400 \mathrm{~m}$ ) in butterfly configuration are distributed over the field. 610 wells were drilled at end 2009 and it is plan to culminate at $\sim 2000$ wells at license end.

The geology features two distinct environments with amalgamated channel fill units at the base and more marine influenced with regional flooding surfaces higher up in the oil column. This will be briefly described since it was key in the definition of the development strategy and during the reservoir management phase.

At this stage of the development, EOR implementations are planned and pilot projects are being launched with thermal mechanisms (SAGD/HASD/SD) and Chemical EOR (polymer sweeping). These Field Tests will be described.

\section{Reservoir development and management}

This may sound as a paradox but slow production mechanism and limited Recovery reached In HO / EHO require early and thorough understanding of the reservoir behavior and response.

As a matter of fact, Heavy Oil requires exacerbated reservoir management discipline since the slow increase in reservoir investigation associated with a slow / uncertain reversibility of the mechanisms leaves very small margin for maneuver in case adjustments are required.

This low flexibility is typically combined with very large volume in place and well intensive developments. This puts even more emphasis on the reservoir development optimization.

Examples of the reactivity that was required at several stages will be shown (well architecture, water ingress understanding and management, surface installations,...)

Reservoir Management as a key to valorize data acquisition and monitoring efforts

Reservoir and production monitoring are therefore crucial since they are key for the production optimization and reserves maximization: a fairly large array of monitoring techniques is required. However, the Risks and Uncertainties need to be clearly identified very early in order to tailor the monitoring to the stakes identified. 
The presentation will detail the typical monitoring required in this mobile HO environment associated with the specific needs of the different possible production mechanisms based on actual field implementations experience. Several monitoring application examples will be described more specifically (Fiber Optic, 4D, Tracers, saturation logging, MPFM,...).

\section{Concluding remarks}

Oil and Gas industry relies on our ability to produce efficiently the Heavy Oil and Extra Heavy Oil resources. Reservoir monitoring and Management are the key to success.

Monitoring to be implemented for HO / EHO recovery is now fairly well mastered but maintaining a sustainable effort is mandatory to durably improve recovery and production.

As for EOR recovery mechanisms, the performance strongly depends on the mechanism implemented and the main hurdle related to maturity of the selected mechanisms will be overcome through dedicated R\&D effort and / or pilot applications. 